\title{
Study of Supratentorial Tumours by Computed Tomography
}

\author{
Prashanth G Patil ${ }^{\circ}$, KM Nataraj ${ }^{\circ}$, Shaik Hussain Saheb ${ }^{\circledR 3}$ \\ ${ }^{1}$ Associate Professor, Department of Radiology, Akash Institute of Medical Sciences \& Research Centre, Devanahalli, Bangalore, Karnataka, India, ${ }^{2}$ rrofessor, \\ Department of Surgery, JJM Medical College, Davanagere, Karnataka, India, ${ }^{3}$ Assistant Professor, Department of Anatomy, JJM Medical College, Davanagere, Karnataka, \\ India.
}

\section{Abstract}

Background: Brain neoplasms may be classified by the location of supratentorial, infratentorial and midline tumours. Of the supratentorial neoplasms, meningiomas are the most frequent extra-axial neoplasms. CT has become the most important diagnostic procedure in evaluating patients suspected of harboring an intracranial tumor. It is still considered the basic radiologic study since it gives specific information for the management of brain tumours and is minimally invasive. The purpose of this study was to assess the distribution, features, localization and extent of supratentorial neoplasms. Subjects and Methods: Fifty cases with symptoms of intracranial pathology and on CT found to have supratentorial tumours were studied. Results: The CT patterns of 50 supratentorial tumours were reviewed, out of which 30 cases i.e. $60 \%$, were found to be intra- axial and 20 i.e. $40 \%$ extra-axial tumours. GBM formed the major group of the intra axial tumours i.e. $18 \%$, and meningiomas formed the major extra-axial tumours forming $26 \%$. Conclusion: CT proves to be a valuable modality of imaging in evaluating the distribution, features, localizing and assessing the extent of various intra and extra-axial tumours in the supratentorial region.

Keywords: Supratentorial Neoplasms, Brain Neoplasms, Tomography, X-Ray Computed, Glioma, Meningioma.

Corresponding Author: KM Nataraj, Professor, Department of Surgery, JJM Medical College, Davanagere, Karnataka, India. E-mail: anatomyshs@gmail.com

Received: 09 March 2020

\section{Introduction}

The concept of a tumor of the brain is, for most individuals and many physicians as well, one of the most dramatic forms of human illness. A brain tumor occurs the second most common form of malignancy in children and primary brain tumours rank from 6th to 8th in the frequency of all neoplasms in the adult. ${ }^{[1]}$ The annual incidence of primary intracranial neoplasms is estimated to be 12.3 persons per 1 lac population and it is increasing in frequency. Since the majority of these tumours present with nonspecific complaints such as headache, strokes like syndromes, or seizures, often a diagnosis is made or suggested initially by the findings on imaging studies. However prognosis of these patients has improved considerably due to recent advances in diagnostic techniques, microsurgery and radiotherapy. Clinical evaluation, radiology and pathology play big roles in deciding the long-term prognosis. Radiological diagnosis is based on the topography of the lesion, characterization into intra V/s extra parenchymal location, morphology analysis and presence of secondary changes adjacent to the lesion. Computed tomography remains the most widely used form of neuroimaging for the diagnosis of brain tumours due to its wider availability and lower cast. Recent advances in imaging techniques have exploded into the horizon of using many different modalities like MRI and CT perfusion, PET and SPECT. These imaging modalities have revolutionized the diagnosis and management of brain tumours. The present study objectives were to study the distribution of various supratentorial neoplasms, to study CT features of supratentorial neoplasms, and to localize and assess the extent of supratentorial neoplasms. ${ }^{\text {[2] }}$

\section{Subjects and Methods}

This study of evaluating the efficacy of computed tomography in the diagnosis of supratentorial tumours was done on 50 cases. The patients who were referred to the department of Radio-diagnosis after suspected to have brain tumours by clinicians are taken up for radiological evaluation and try to detect by CT scan. The study was conducted in the Department of Radiodiagnosis. Thorough clinical history and clinical examination were made before CT examination. The data were analysed and expressed in percentage. 


\section{Results}

Primary cerebral gliomas are the largest group of all intracranial tumours. Our study encompassed 23 cases of gliomas which included 5 low-grade gliomas that is $21.7 \%$ of the gliomas. We had 3 males and 2 females. The commonest location was the frontal lobe (i.e. 3 cases) and in the other 2 cases, it was found in the parietal and temporal lobe, respectively. On NECT the lesions had irregular borders and minimal edema in one case. On CECT 2 out of 5 cases showed slight contrast enhancement. On NECT, the infiltrating pattern of the lesions could be seen with obliteration or compression of subarachnoid spaces. Tumor margins were blurred in 2 of the cases located in the frontal lobe which showed extension into the contralateral hemisphere, indicating the infiltrating nature of neoplasm.

In our study, out of 23 cases of gliomas, 15 were a high grade that is $65 \%$. Out of which 6 were anaplastic type and 9 of GBM type. The mean age in our study was between 51-60 years with a male preponderance. We had 7 patients having frontal lobe predominance out of 15 cases. On NECT there was 1 patient who had calcification. On CECT, the bulky enhancing mass was seen in $6.6 \%$ of our study and 2 patients showed exophytic growth. In our study, there was a case of GBM showing an ill-defined infiltrating pattern involving the deep white matter in the right frontal region and extending across the corpus callosum into the opposite hemisphere and another case on CECT showed GBM with a medial extension of the neoplasm into the corpus callosum with a so-called butterfly component.

Out of 23 cases of gliomas, we found 1 case of oligodendroglioma in a man presenting with a history of seizures and headache showed a hypodense lesion in the subcortical region of the frontal lobe with discrete calcification on NECT. On CECT there was slight homogenous enhancement with tumor extension into the cortex. In our study of 49 cases of primary intracranial neoplasms, we found one case of ependymoma that is $2 \%$ of all primary intracranial neoplasms. There was a 5-year-old male child with a history of headache and ataxia showing a hypodense solid mass located in the periventricular region, moderately marginated and showed moderate homogenous enhancement on CECT with minimal edema and no hemorrhage or calcification with evidence of extension noted to the $\mathrm{CP}$ angle cisterns.

In our study of 49 cases of primary intracranial neoplasms, we found one case of subependymoma that is $2 \%$ of all primary intracranial neoplasms. There was an elderly male of 60 years presenting with a history of headache showing a hypodense lesion of cystic consistency located in the lateral ventricle, causing hydrocephalus due to tumor extension.

In our study, we had 2 cases of primary CNS lymphoma (i.e. $6.6 \%$ ) of intra axial tumours and of $4 \%$ of the total intracranial tumours. We had 2 patients, one 57 years old and the other 64 years presenting with a history of headache and convulsions showing a hyperdense lesion in the basal ganglia and thalamus. Post-contrast studies showed a single nodular lesion and thickwalled ring enhancement with significant perilesional edema. Tumour was extending subependymal. The other case of a 64-year-old man showing multiple isodense lesions located in the parietal lobe at cortico-medullary junction showed intense homogenous enhancement on CECT. No evidence of extension noted to other sites.

We found one case of primary cerebral neuroblastoma forming $3.3 \%$ of intra axial tumours and $2 \%$ of the intracranial tumours. There was a male child of 6 years old showing an ill-defined, well-circumscribed hypodense lesion, cystic in consistency located in the frontal lobe showing discrete calcification. On CECT there was intense homogenous enhancement seen with areas of necrosis noted within, with minimal perilesional edema. No evidence of extension noted to other sites. We had one case of germinoma forming $2 \%$ of intracranial and $3.3 \%$ of intra-axial tumours. There was a case of a 12 years old male child with a history of headache showing a hyperdense lesion in the posterior end of 3rd ventricle with evidence of calcification on the pre-contrast study. On post-contrast study, there was intense enhancement seen. Tumour was seen extending into the 3 rd ventricle, causing hydrocephalus.

In our study, metastasis formed $6 \%$ of all the intracranial neoplasms, where we found 3 cases, i.e.3.3\% of intra axial tumours. All our 3 cases were males within an age range of 5170 years. Most common symptoms were seizures, headache and ataxia. Two patients had metastasis in the frontal lobe and one in the parietal lobe. On NECT. 2 were isodense and one was hypodense and on CECT, 2 cases showed slight enhancement and one moderate enhancement. Necrosis was seen in one case. There was moderate edema in all 3 cases.

We had 13 cases of meningiomas in our study, which constituted $65 \%$ of extra-axial tumours and $26 \%$ of all intracranial tumours. There was a female preponderance. Out of 13 cases, there were 9 females and 4 males. The most common age group was in the 50-70 years age group (i.e.7/13). Nine cases presented with either hemiplegia or hemiparesis and 1 case presented with vertigo and tinnitus. Two cases presented with either blindness or visual blurring and 2 cases presented with ataxia. The cerebral convexity was the most common location, accounting for 7 cases. The next common was parasagittal accounting for 5 cases.

On NECT scan, the most common appearance was a welldefined rounded hyperdense lesion with perilesional edema, seen adjacent to either bone or falx. Seven cases were hyperdense, 3 cases were isodense and 3 cases were of mixed density. No cases of hypodense lesions were recorded. Calcification was seen in 5 cases. Three cases showed either bone destruction or bone hyperostosis. Necrosis was seen in one case. Following IV contrast administration, the extra-axial 
features were more prominent, the broad base to bone or falx was seen in all most all the cases. Nine cases showed intense, homogenous enhancement of more than $40 \mathrm{HU}$, while 4 cases showed moderate enhancement and only one case showed mild enhancement of around $20 \mathrm{HU}$. We recorded 1 case of multiple meningiomas; occurring in a female. In the other case in a female aged 60 years presented with headache had lesioned at the left sphenoid ridge and left parietal convexity. Both lesions showed dense areas of calcifications. There was no perilesional edema. We studied 5 cases of pituitary adenomas, constituting $25 \%$ of all extra-axial tumours and $10 \%$ of all intracranial tumours. The commonest presenting symptom was blindness or visual blurring found in 4 out of 5 cases studied. Three cases presented with headache and 1 case presented with convulsions.

A male predominance was noted in the ratio of 3:2. Three cases seen in males and 2 cases in females. Two cases were found in less than 25 years of age group and 2 cases in 25-50 years age group and 1 case in the fifth decade. In our series, we had 4 cases of macroadenoma and 1 case of microadenoma. Out of the five cases studied, 2 cases were hyperdense, 2 cases were isodense, 1 case was of mixed density on a plain scan. One case showed areas of hemorrhage and 1 case showed calcification in the periphery of the tumor. On contrast administration, all cases showed a periphery of the tumor. In contrast, the administration of all cases showed intense enhancement. Two cases were enhancing homogenously and 2 cases heterogeneously. All five cases showed well-defined margins, with a broad base towards sella. All 4 cases of pituitary macroadenoma showed a widening of sella turcica. One case of pituitary microadenoma was seen in a 45-year-old female who presented with nonspecific headache.

We recorded 2 cases of craniopharyngiomas, one case occurring in female aged 14 years and other in a 55-year-old male. Both cases presented with headache and visual blurring. Both the cases showed a mixed density lesion with nodular calcified areas. In one case i.e., in 14-year-old females showed enhancement of peripherally situated solid nodule. In both cases lesion was seen in the sellar and suprasellar region. Calcification was seen in both cases. Sellar erosion was seen in one case.

\section{Discussion}

The differential diagnosis of intracranial lesions begins with an accurate assessment of the lesion location. In fact, the most important diagnostic feature of an intracranial mass is its location. Intracranial masses are commonly divided into intraaxial and extra-axial locations. ${ }^{[3]}$ Primary cerebral gliomas are the largest single group of all intracranial tumours. ${ }^{[4]}$ Baker et al out of 1,071 intracranial neoplasms reported an incidence of $34 \%$ for gliomas, of which $28.38 \%$ were supratentorial. ${ }^{[5]}$ In our study gliomas represented $46 \%$ of all primary intracranial tumours and $76 \%$ of all intra axial tumours in the supratentorial region. According to Leeds, NE et al of 100 cases of supratentorial gliomas, included 29 lowgrade gliomas i.e. $29 \%{ }^{[6]}$ Our study encompassing 23 cases of supratentorial gliomas included 5 were low-grade gliomas i.e. $21.7 \%$. A study by Butler AR et al among 14 cases of lowgrade glioma, 9 were males and 5 females. ${ }^{[7]}$ Our study of 5 patients of low-grade glioma we had 3 males and 2 females i.e. $60 \%$ suggesting male preponderance as per the study done by Butler AR et al. ${ }^{[7]}$ According to a study by Butler AR et al median age of patients with low grade glioma was 38 years correlating with our study with age range lying between 21 40 years (3/5). ${ }^{[7]}$ In our study we had 3 out of 5 cases having a primary location of the lesion in the frontal lobe which is $60 \%$ as per the study on 14 cases of low-grade glioma, 10 patients had a primary location in the frontal lobe which is $71 \%$ as per the study done by Butler AR et al. ${ }^{[7]}$ According to Leeds NE et al an important feature of grade I and II gliomas on NECT is the presence of well-demarcated lesions with moderately sharp borders and little or no edema. IV contrast enhancement occurs less frequently in low-grade gliomas as per the study it was seen in $48 \%(14 / 29) .{ }^{[6]}$ In our study 2 out of 5 cases showed contrast enhancement i.e. $40 \%$ which is of little variance compared to the above study. Most of the cases in our study had irregular borders with minimal edema. Our study showed $60 \%$ hypodense and $40 \%$ isodense lesions. Our study correlated well with the study done by Leeds NE et al (1984). About $40 \%$ showed some amount of enhancement while the others didn't show any enhancement which correlated well with the study done by Butler AR et al, ${ }^{[7]}$ where they found $37 \%$ out of 14 cases of low-grade gliomas showing slight enhancement (3/14).

According to Butler AR et al, ${ }^{[7]} 46$ cases out of 60 cases were high-grade gliomas i.e. $76 \% .43$ In our study out of 23 cases of gliomas, 15 were high grade i.e. $65 \%$ out of which, 6 were Anaplastic type and 9 of Glioblastoma multiforme (GBM) type i.e. $26 \%$ and $39.1 \%$ respectively. According to Butler AR et $\mathrm{al}^{\left[{ }^{[7]}\right.}$ the mean age was 58 years with a male predominance 43 which correlated well with our study, mean age being between [51-60] years with a male predominance (9/15). In a study done by Butler AR et al, ${ }^{[7]}$ the primary site of HGG (highgrade glioma) was frontal lobe (22/46) i.e. $47.8 \%$. In our study we had 7 patients with frontal lobe predominance out of 15 cases, which is $46 \%$ correlating well with the study done by Butler et al. According to Leeds NE of the 100 patients, ${ }^{[6]}$ 71 were high-grade glioma, all were found to have contrast enhancement. As per our study done on 23 cases of gliomas, 15 were high-grade glioma, of which all were having contrast enhancement. Opacification in the more malignant tumours is related to vascularity necrosis, pleomorphism and cellularity. According to Leeds NE et al, ${ }^{[6]}$ of the 57 high grade gliomas with unilocular or multilocular rings on CECT, 30 showed no 
rings on NECT while 19 had an incomplete ring and only 8 had complete ring lesion. On NECT, calcification was observed in 4 patients (i.e. 5.6\%). A nodular mass with opacification occurred in 14 patients (i.e. 19.7\%). A bulky enhancing mass was visualized in 8 patients (i.e. 11.2\%). Exophytic growth of neoplasms to a meningeal surface was noted in 6 cases (i.e. $10.3 \%){ }^{[6]}$ In our study, out of 10 cases of high-grade gliomas showing unilocular or multilocular rings on CECT, 6 showed no rings on NECT while 3 had an incomplete ring and only 1 a complete ring lesion. On the NECT, there was only 1 patient who had calcifications (i.e. 6.6\%) nodular mass with opacifications was seen in 2 patients (i.e. 13.3\%). Bulky enhancing mass was seen in $6.6 \%$ of our study and 2 cases showed exophytic growth (i.e. 13.3\%). Our study correlates well with the study done by Leeds NE et al. ${ }^{[6]}$

In a study done by Birjandi A (1981-2000) found 35 cases of oligodendroglioma i.e. $7 \%$ of total gliomas. ${ }^{[8]}$ In our study out of 23 cases of gliomas, we found 1 case of oligodendroglioma i.e. $4.3 \%$ out of all gliomas. According to Birjandi $\mathrm{A}$ in among 35 cases of oligodendrogliomas, 27 were males $(77.1 \%)$ and 8 females $(22.9 \%)$ ranging in the age group of 4 to 68 years (mean age 36.9 years). ${ }^{[8]}$ Seizures and headache were present in 20 patients (i.e. $57 \%$ ), vomiting and papilledema in 15 patients (i.e. $43 \%$ ) and hemiparesis in 12 patients (i.e.28.8\%). ${ }^{[8]}$ We had a case of oligodendroglioma in a 46-year-old man with a history of headache and seizures. According to Birjandi A lesions involved frontal lobe in 20 patients (i.e.57.12\%), Temporal lobe in 8 patients (i.c. $22.88 \%$ ), parietal lobe 5 in patients $(14.3 \%$ o) and multiple lobes in 2 patients (i.e.5.7\%) calcifications were present in 25 tumours (i.e.71.4\%). Most of the tumours were iso to hypodense and most did not show contrast enhancement. In our study on NECT there was a hypodense lesion noted in the right frontal lobe with evidence of calcifications and on CECT showed moderate heterogenous enhancement. Our study correlates well with the study done by Birjandi A. ${ }^{[8]}$

According to Osborn, AG et al. found $0.3 \%$ and $0.6 \%$ of ependymoma in children and young adults, respectively. In our study, out of 29 cases of primary intracranial neoplasms, we found one case of ependymoma i.e. $2 \%$ of all primary intracranial neoplasms which correlates well with the study done by Osborne AG. According to Osborn AG et al. of 16 cases of ependymoma, $63 \%$ were males and $37 \%$ were females. In our study conducted, we had a 5-year-old male child with a history of headache correlates well with the study done by Osborne AG (1985). According to Osborn $\mathrm{AG}$ et al. of 16 cases, $19 \%$ were periventricular located, $25 \%$ had solid consistency, $56 \%$ low density on NECT and $43 \%$ were moderately enhancing on CECT of which $38 \%$ showed homogenous enhancement with no hemorrhages, $38 \%$ calcification and 56\% edema. Our study showed a hypodense lesion located periventricular with no calcification, moderately marginated showing moderate, homogenous enhancement with minimal edema and no hemorrhage noted. Our study correlates well with a study done by Osborne AG. ${ }^{[9]}$

A rare variant of ependymoma that actually consists of both highly differentiated ependymal cells and astrocytes. According to Jones RV et al. on 24 patients with pathologically proven cases of intracranial subependymoma, an age range of (39-59 years) with $72 \%$ incidence among males (17/24), the headache was the commonest symptom. In our study, out of 49 cases of primary intracranial neoplasms, we found one case of subependymoma which is $2 \%$ of all primary intracranial neoplasms. Out study constituted an elderly 64-year-old male presenting with history of headache. According to Jones RV et al. on 24 patients with proven intracranial subependymoma, approximately $60 \%$ occurred in the lateral ventricle and $40 \%$ occurred in the $4^{\text {th }}$ ventricle correlating with our study where we had a hypodense lesion of cystic consistency located in the lateral ventricle causing hydrocephalus. According to Jones RV et al. on 24 patients of proven intracranial subependymomas, 14 cases were supratentorial in origin. Eleven out of which were hypodense on NECT were located in lateral ventricle subependymomas, whereas the 4 th ventricular subependymomas were variable in appearance. One out of 14 subependymomas had calcifications on NECT. On CECT, all had minimal, discrete enhancement. In our study, we had a hypodense lesion located in the lateral ventricle, cystic in consistency showing minimal heterogeneous enhancement on CECT. ${ }^{[10]}$

Primary lymphoma of the CNS is a rare tumor, representing $0.2 \%-2 \%$ of all primary CNS malignancies. According to Jack CR et al. (1972-1984) on 32 patients with pathologically proven primary CNS lymphoma (21 i.e. $65 \%$ males and 11 i.e. $34 \%$ females) ages were $9-79$ years (average being 58.8 years). In our study we found 2 patients of primary CNS lymphoma i.e. $6.6 \%$ of the intra-axial tumours and $4 \%$ of total intracranial tumours in which both were males with age between (57-70) and meant age 60.5. Our study matched well with the study done by Jack CR et al. According to Jack CR et al. (1972-1989) on 32 patients of pathologically proven primary CNS lymphoma found 19 of 32 lesions hyperdense (i.e. $59.3 \%$ ). $30 \%$ of lesions were located in deep central structures, $46 \%$ peripherally and $13 \%$ in the posterior fossa. In our study of 2 cases of primary CNS lymphoma, we found one hyperdense lesion located in the basal ganglia and thalamus and the other isodense lesion in the parietal lobe, located at the corticomedullary junction. Our study correlates well with the study done by Jack CL et al. According to Jack $\mathrm{CR}$ et al. on CHCT, all lesions showed enhancement and 17 showed homogenous enhancement i.e. 53\%. They had a higher incidence of ring enhancement. The enhancing wall of tumor nodule was thick. A prominent peritumoral edema zone was common in their series (26/32). In our study we had both 
cases showing enhancement one with single nodular lesion and thick-walled ring enhancement and the other showed intense homogenous enhancement. ${ }^{[11]}$ In a study, Spillane et al found subependymal spread in 3 of 20 cases, whereas we found only 1 of the 2 cases showing subependymal spread. ${ }^{[12]}$

Primary cerebral neuroblastomas are rare tumours of childhood and early adolescence. In a study, Chamber et al. on 9 patients of biopsy proved cases of supratentorial, primary cerebral neuroblastomas involving the brain parenchyma found these tumours most often occurred in childhood and early adolescence and had no sex predilection. In our study we found one case of primary cerebral neuroblastoma in a 6-year-old male child forming $3.3 \%$ of intraaxial tumours and $2 \%$ of the intracranial tumours correlating well with the study done by Chambers et al. In a study conducted by Chamber et al on 9 cases of PNET, frontal lobe was the commonest location, (5/9) were hyperdense (i.e.55.5\%), 2 were isodense and 2 were between isodense to hypodense i.e. $22.2 \%$ each and gross calcifications were noted in 5 cases (i.e.55.5\%). On CECT, enhancement was seen in all cases (9/9), low-density zones in (7/9), cystic (3/9) and necrotic (4/9) with peritumoral edema in almost all cases (8/9). In our study, there was a case of PNET showing an ill-defined, well-circumscribed hypodense lesion, cystic in consistency located in the frontal lobe showing discrete calcifications on post-contrast study there was intense homogenous enhancement seen with areas of necrosis noted within with peritoneal edema. Our study matched well with the study done by Chamber et al. ${ }^{[13]}$

Most intracranial metastasis appears to reach the brain via the arterial bloodstream, but some may extend to the surface of the brain. According to Potts et al in a series of 313 patients with intracranial metastasis, $76 \%$ of the metastases occurred in patients who were between 41 and 70 years of age. They were more common in males than in females (171:142). The most common symptoms were headache (43\%), gait problems (37\%) and cranial nerve abnormalities (33\%). Our study metastasis formed $6 \%$ of the intracranial neoplasms, where we found 3 cases, i.e. 3.3\% of intraaxial tumours. All our 3 cases were males with an age range between 51-70 years, with symptoms of seizures and headache and ataxia seen in 1 patient. Our study matched well with the study done by Potts et al. According to Potts et al. study on 313 patients, CT features of the brain showed the most frequent sites involved were the frontal region, parietal region and posterior fossa. In our study of 3 cases, 2 patients had locations in the frontal lobe and 1 parietal lobe correlating well with the study done by Potts et al. ${ }^{[14]}$ According to Potts et al. on NECT the tumor density was equal to or greater than the surrounding brain with attenuation values ranging up to $40 \mathrm{MU}$. On CECT, necrosis was seen in $90 \%$ of all lesions and contrast enhancement was $20-40 \mathrm{HU}$ above and nearly all cases showed edema. In our study of 3 cases of metastasis, we had 2 with isodense lesions, 1 was hypodense and on post-contrast study, 2 cases showed slight enhancement and 1 moderate enhancement necrosis was seen in 1 patient. There was moderate edema noted in all 3 cases which correlated well with the study done by Potts et al. ${ }^{[14]}$

According to Packer et al study on 25 patients of pineal tumor who underwent biopsy 8 were (pineoblastomas, pineocytomas) i.e. $32 \%, 8$ were germ cell tumours (germinomas) i.e. $32 \%$ and 8 were glial tumours (astrocytomas, gangliogliomas) i.e. $32 \%$ and ganglioneuroblastoma in one patient $(4 \%){ }^{[15]}$ According to Fujimaki et al, $80 \%$ of intracranial germinoma is found in the pineal region and the remaining $20 \%$ are usually suprasellar.52 In our study, there was 1 case forming $3.3 \%$ of total interracial tumours and $2 \%$ of entire intracranial tumours. The lesion was located in the pineal region, correlating well with the study done by Fujimaki et al. ${ }^{[16]}$ Rushing et al. studies found a strong male preponderance with age range between (10-30 years). ${ }^{[17]}$ In our study there was a 12 -year-old male child with a history of headache showing the tumor. According to Weisberg LA in 60 histologically proven tumours of germinoma, ${ }^{[18]}$ on NECT found them appearing as hyperdense lesions in the posterior 3rd ventricular region and showed homogenous enhancement which sometimes extends to the anterior 3rd and lateral periventricular region with areas of calcifications. In our study of 1 case of germinoma, there was a hyperdense well-marginated lesion noted posterior to 3rd ventricle with evidence of calcifications and showed homogenous enhancement on CECT. The tumor extended into the anterior part of $3^{r d}$ ventricle. Our study correlated well with study done by Weisberg. [18]

According to Curnes JT meningioma is the most common primary non-glial intracranial neoplasm, representing $15 \%$ of all primary brain tumours. ${ }^{[19]}$ Zimmerman in a study of 2262 intracranial neoplasms, reported an incidence of $27.3 \%$ for meningiomas. ${ }^{[20]}$ In our study, meningioma represented $26 \%$ of all primary intracranial tumours and $65 \%$ of all extraaxial tumours with a female sex preponderance, as noted in most of the studies. According to Paul FJ et al in their study of 164 cases showed maximum incidence in the $6^{\text {th }}$ and $7^{\text {th }}$ decade. In our study 7 cases i.e. $53.8 \%$ of the tumor occurred in the $6^{\text {th }}$ and $7^{\text {th }}$ decade. Three cases in the $7^{t h}$ decade and 4 cases in $6^{\text {th }}$ decade. ${ }^{[21]}$ According to Rohinger et al. study in a population-based study of 193 patients with intracranial meningioma, found headache and paresis to be the most common symptoms and sign in $36 \%$ and $30 \%$ of patients, respectively. In our study $53.8 \%$ of cases presented with headache, $38.4 \%$ of cases presented with seizures and hemiplegia. This is of little variance from a study done by Rohinger et al All the tumor had a broad base to either bone, falx or tentorium. Meningiomas generally appear as well ascircumscribed lobular lesions. Most have homogenously high density. ${ }^{[22]}$ According to Sutton et al., on NECT about $60 \%$ of all meningiomas are usually-homogenous well-circumscribed 
hyperdense solid masses and calcification is seen in upto $20 \%$ of the cases. ${ }^{[23]}$ In our study, 57\% were hyperdense, $23 \%$ isodense and $27 \%$ were mixed density on a precontrast scan. Our study correlated well with that of Sutton et al. About 73\% showed homogenous enhancement of which $53.8 \%$ showed intense enhancement of more than $40 \mathrm{HU}$ while moderate enhancement in the range of $20-40 \mathrm{HU}$ was seen in $15.3 \%$. The degree of enhancement is dependent both on tumor vascularity and extracellular accumulation of contrast. ${ }^{[23]}$

Calcification in meningiomas occurs in the form of psammomatous calcification or patchy nodular calcification. It is not always possible to differentiate between these two forms of calcification with confidence in CT. According to Buetow MP et al calcification is seen in about $20-25 \%$ cases in our study calcification was seen in $38.4 \% .{ }^{[24]}$ Edema is seen as low density within the white matter adjacent to the tumor. Occasionally edema may spread throughout the hemispheric white matter. It may be noted that low-density regions around meningiomas may represent encephalomalacia rather than vasogenic edema per se, and this zone can also include an extension of the subarachnoid space or a cyst around the tumor (one of the features to suggest the extra-axial location of a lesion). Patients receiving steroid therapy may have altered perilesional edema. According to Buetow MP et al, ${ }^{[24]}$ perilesional edema was seen in $60 \%$ of meningiomas. In our study, perilesional edema was noted in about $46.6 \%$ meningiomas. This is of little variance from that of the Buetow MP et al. study. ${ }^{[24]}$ According to Nadich TP et al hyperostosis (bone sclerosis) for bone destruction is seen in approximately $15-20 \%$ of meningiomas. ${ }^{[25]} \mathrm{In}$ our study bony changes were seen in $13.2 \%$.

Hyperostosis is generally thought to be due to invasion of the overlying bone by the meningioma cells. Bone destruction from meningiomas occurs either because of the infiltration of bone by the tumor or from pressure erosion of the overlying bone from an adjacent slow-growing meningioma. According to Russel JE et al. areas of hyperdensity, hypodensity, and non-uniform enhancement may be seen and may represent hemorrhage, cystic degeneration or necrosis. ${ }^{[26]}$ In our study, necrosis was seen in $7.6 \%$ of meningiomas. According to Nadich TP et al. frequent sites of origin include the frontal and parietal convexities and parasagittal regions (about 45\%) as well as the sphenoid wing $(15.20 \%)$ para sellar $(5-10 \%)$. Less than $10 \%$ arise below the level of the tentorium. Multiple meningiomas occur in $6-9 \%$ of cases. ${ }^{[25]}$ In our study convexities and parasagittal regions, meningiomas constitute 92.2\% and sphenoid ridge constitutes $7.6 \%$.

According to Kovac SK et al. pituitary adenomas are common lesions, account for approximately $10-15 \%$ of all primary intracranial and between one third and one-half of all sellar/juxtasellar masses. In our study they constitute about $10 \%$ of all intracranial neoplasm and $25 \%$ of all extra-axial tumours. According to Kovac SK et al. pituitary adenomas are mainly tumours of adulthood, with less than $3 \%$ occurring in individuals younger than 18 years. In our study 4 out of 5 cases i.e. $80 \%$ of cases occurred in the adult age group with no cases reported less than 18 years of age. ${ }^{[27]}$

Pituitary microadenomas are tumours less than $10 \mathrm{~mm}$ in diameter. Microadenomas uncomplicated by hemorrhage or cyst formation are typically isodense with the adjacent normal pituitary gland and may be invisible on NECT scans. Enhancement following contrast administration occurs but is usually delayed compared to the immediate, intense enhancement of the normal pituitary gland. Our single case of pituitary microadenoma was isodense with the normal pituitary gland. A contrast study showed intense and homogenous enhancement. According to Rao Krishna VG et al., pituitary macroadenomas normally show homogenous density similar to or slightly greater than that of normal brain tissue and enhance uniformly after contrast administration. Intra tumoral necrosis, cystic changes and hemorrhage all can occur more frequently in macroadenomas than microadenomas. Hemorrhage may be present in $20-30 \%$ of macroadenomas. ${ }^{[28]}$ Our study showed hemorrhage in $20 \%$ of cases and peripheral calcification in $20 \%$ of cases. Almost all cases showed intense enhancement on contrast scan. Our study correlated with Rao Krishna CV et al. study. ${ }^{[28]}$

This tumor arises from the epithelial cell rests along the involuted- hypophyseal Rathke's duct. According to Fitz RC et al, ${ }^{[29]}$ craniopharyngiomas account for $3 \%$ of all tumours in all age groups. In our study, it accounted for $4 \%$. According to Osborne AG more than half of craniopharyngiomas occur in children and younger adults. $40 \%$ of craniopharyngiomas in children occurred between 8 and 12 years of age. A second somewhat smaller peak occurs in middle-aged adults. There was no sex predilection. In our study of 2 cases one case occurred in 14 years old female and other in 55-year-old male. According to Fitz RC et al. features of craniopharyngioma are contrast enhancement, calcification and cyst formation. A combination of at least two of these three signs in a centrally situated suprasellar tumor seen a reliable indicator of a craniopharyngioma. Nodular calcification is present in nearly all pediatric craniopharyngiomas and is identified in $50 \%$ of adult cases. In our study, the solitary case of pediatric craniopharyngioma showed nodular calcification. Tumor contents are slightly higher in attenuation than CSF. Post-contrast study can demonstrate nodular or rim enhancement in more than $90 \%$ of all cases. One of the cases in our study showed nodular peripheral enhancement. CT is a valuable tool with high diagnostic sensitivity and prognostic accuracy in the evaluation of supratentorial tumours. In the armamentarium of non - invasive techniques, CT becomes the mainstay of investigation from the viewpoint of accuracy, safety and cost-effectiveness. CT scan findings assume great diagnostic significance in supratentorial tumours, when considered in conjunction with clinical findings. 


\section{Conclusion}

CT proves to be a valuable modality of imaging in evaluating the distribution, features, localizing and assessing the extent of various intra and extra-axial tumours in the supratentorial region.

\section{References}

1. Bondy ML, Scheurer ME, Malmer B, Barnholtz-Sloan JS, Davis FG, Il'yasova D. Brain Tumor Epidemiology: Consensus from the Brain Tumor Epidemiology Consortium (BTEC). Cancer. 2008;113((7 Suppl)):1953-1968. Available from: https://dx.doi.org/10.1002/cncr.23741.

2. Patibandla MR, Bhattacharjee S, Uppin MS, Purohit AK. Outcome of supratentorial intraaxial extra ventricular primary pediatric brain tumors: A prospective study. J Pediatr Neurosci. 2014;9(3):216-220. Available from: https://dx.doi.org/10. 4103/1817-1745.147571.

3. Lyndon D, Lansley JA, Evanson J, Krishnan AS. Dural masses: meningiomas and their mimics. Insights Imaging. 2019;11:122. Available from: https://doi.org/10.1186/s13244-019-06977.

4. Singh H, Maurya V, Gill SS. Computerised Tomography Features in Gliomas. Med J Armed Forces India. 2002;58(3):221-225. Available from: https://dx.doi.org/10. 1016/S0377-1237(02)80134-4.

5. Baker HL, Houser OW, Campbell JK. Baker HL National cancer institute study. Evaluation of Computed tomography in the diagnosis of intracranial Neoplasms. Radiology. 1980;136(1):91-96. Available from: https://doi.org/10.1148/ radiology.136.1.7384529.

6. Leeds NE, Elkin CM, Zimmerman RD. Gliomas of the brain. Seminars Roentgenol. 1984;19(1):27-43. Available from: https://dx.doi.org/10.1016/0037-198x(84)90041-5.

7. Butler AR, Passalaqua AM, Berenstein A, Kricheff II. Contrast enhanced CT scan and radionuclide brain scan in supratentorial gliomas. Am J Roentgenol. 1979;132(4):607-611. Available from: https://dx.doi.org/10.2214/ajr.132.4.607.

8. Birjandi A. Oligodendrogliomas A report of 35 cases. Iranian J Otorhinolaryngol. 2005;17(41):19-25.

9. Armington WG, Osborn AG, Cubberley DA, Harnsberger HR, Boyer R, Naidich TP, et al. Supratentorial ependymoma: CT appearance. Radiology. 1985;157(2):367-372. Available from: https://doi.org/10.1148/radiology.157.2.4048443.

10. Chiechi MV, Smirniotopoulos JG, Jones RV. Intracranial subependymomas: CT and MR imaging features in 24 cases. Am J Roentgenol. 1995;165(5):1245-1250. Available from: https://dx.doi.org/10.2214/ajr.165.5.7572512.

11. Jack CR, Reese DF, Scheithauer BW. Radiographic findings in 32 cases of primary CNS lymphoma. Am J Roentgenol. 1986;146(2):271-276. Available from: https://dx.doi.org/10. 2214/ajr.146.2.271

12. Spillane JA, Kendall BE, Moseley IF. Cerebral lymphoma: clinical radiological correlation. J Neurol Neurosurg Psychiatry. 1982;45(3):199-208. Available from: https://dx.doi.org/ 10.1136/jnnp.45.3.199.
13. Chambers EF, Turski PA, Sobel D, Wara W, Newton TH. Radiologic characteristics of primary cerebral neuroblastomas. Radiology. 1981;139(1):101-104. Available from: https://dx. doi.org/10.1148/radiology.139.1.7208909.

14. Baker HL, Houser OW, Campbell JK. National Cancer Institute study: evaluation of computed tomography in the diagnosis of intracranial neoplasms. I. Overall results. Radiology. 1980;136(1):91-6. Available from: https://dx.doi.org/10.1148/ radiology.136.1.7384529.

15. Parker RJ, Sutton LN, Rosenstoch JG, Bilaniock LT, Zimmerman RA. Pineal region tumours of childwood. Pediatr. 1994;9(3):217-226.

16. Fujimaki T, Mastsumani M, Furada N, Kirino T, Takakura K, Nakamura O, et al. CT and MRI features of intracranial germ cell tumours. Jour Neurooncol. 1994;9(3):217-226. Available from: https://doi.org/10.1007/bf01053275.

17. Smirniotopoulos JG, Rushing EJ, Mena H. Pineal region masses: differential diagnosis. RadioGraphics. 1992;12(3):577-596. Available from: https: //dx.doi.org/10.1148/radiographics.12.3.1609147.

18. Weisberg LA. Clinical and computed tomographic correlations of pineal neoplasms. Computerized Radiol. 1984;8(5):285-292. Available from: https://dx.doi.org/10. 1016/0730-4862(84)90040-4.

19. Curenes JT. MR imaging of peripheral intracranial neoplasms. Extra axial v/s Intraxial masses. J Comput Assis Tomogr. 1987;11(6):932-937. Available from: https://doi.org/10.1097/ 00004728-198711000-00002.

20. Zimmerman HM. Brain Tumors-Their incidence and classification in man and their experimental production. Ann NY Acad Sci. 1969;159(2):337-359. Available from: https://www.researchgate.net/deref/http\%3A//dx.doi.org/10. 1111/j.1749-6632.1969.tb48290.x.

21. Paul EJ. Diagnosis of intracranial neoplasms. Neuroradiology. 1980;66:675-683.

22. Rohringer M, Sutherland GR, Louw DF, Sima AA. Incidence and clinicopathological features of meningioma. J Neurosurg. 1989;71((5 Pt 1)):665-672. Available from: https://doi.org/10. 3171/jns.1989.71.5.0665.

23. Lyndon D, Lansley JA, Evanson J, Krishnan AS. Dural masses: meningiomas and their mimics. Insights Imaging. 2019;11:122. Available from: https://dx.doi.org/10.1186/s13244-0190697-7.

24. Buetow MP, Buetow PC, Smirniotopoulos JG. Typical, atypical, and misleading features in meningioma. Radiographics. 1991;11(6):1087-1106. Available from: https://dx.doi.org/10. 1148/radiographics.11.6.1749851.

25. Watts J, Box G, Galvin A, Brotchie P, Trost N, Sutherland $\mathrm{T}$. Magnetic resonance imaging of meningiomas: a pictorial review. Insights Imaging. 2014;5(1):113-122. Available from: https://dx.doi.org/10.1007/s13244-013-0302-4.

26. Eric RJ, George AE, Irvin KL, Budzlovich G. Atypical CT features of intracranial meningiomas. Radiological Pathological correlation in a study of 131 consecutive cases. Radiology. 1980;135:673-682.

27. Kovas SK, Horvath E, Sl A. Classification and pathology of pituitary tumours. Newyork: Mc Graw-Hill; 1984. 
28. Kunimatsu A, Kunimatsu N. Skull Base Tumors and TumorLike Lesions: A Pictorial Review. Pol J Radiol. 2017;82:398409. Available from: https://dx.doi.org/10.12659/PJR.901937.

29. Fitz R, Charles. CT in Craniophagiomas. . Radiology. 1978;127:687-691.

Copyright: (C) the author(s), 2020. It is an open-access article distributed under the terms of the Creative Commons Attribution License (CC BY 4.0), which permits authors to retain ownership of the copyright for their content, and allow anyone to download, reuse, reprint, modify, distribute and/or copy the content as long as the original authors and source are cited.

How to cite this article: Patil PG, Nataraj KM, Saheb SH. Study of Supratentorial Tumours by Computed Tomography. Asian J. Med. Radiol. Res. 2020;8(1):114-121.

DOI: dx.doi.org/10.47009/ajmrr.2020.8.1.21

Source of Support: Nil, Conflict of Interest: None declared. 